\title{
Capsulectomía química y cierre de la cápsula mamaria con colgajos de anclaje
}

\author{
Chemical capsullectomy and closing of the breast capsule with \\ anchoring flaps
}

\author{
Guillermo Blugerman*, Diego Schavelzon*, Roberto Rodrigo Cáceres**, \\ Victoria Schavelzon**, Miguel Mussi**, Guido Blugerman**.
}

REVISTA ARGENTINA DE CIRUGÍA PLÁSTICA 2018;24(2):67-72. DOI/10.32825/RACP/201802/0067-0072

\section{INTRODUCCIÓN}

Las prótesis de silicona se han utilizado en la reconstrucción y el aumento estético de las mamas desde los años 60. Algunos pacientes que son receptores a largo plazo de estos dispositivos están ahora informando complicaciones que requieren su eliminación. En el momento de la explantación quirúrgica, la cápsula fibrosa existente relacionada con el implante puede ser removida completamente por capsulectomía. Alternativamente, la cápsula puede ser modificada para recibir un implante de reemplazo o puede dejarse en su sitio a medida que se crea una nueva cavidad de implante anterior o posterior al músculo pectoral intermedio. La formación de una cápsula alrededor del implante mamario es un proceso inflamatorio habitual ante la presencia de un cuerpo extraño. La cápsula periprotésica suele estar bien definida a las 3 o 4 semanas de la cirugía. La histología de la cápsula suele ser distinta cuando se utilizan implantes lisos o texturizados, y también es diferente cuando se forma en el espacio retroglandular o en el espacio submuscular. Cuando usamos implante de superficie lisa, la cápsula suele ser delgada, blanquecina o rosada, unida a los tejidos adyacentes y con una superficie interna lisa, brillante y no adherida al implante. En algunas cápsulas de muchos años de evolución se ha observado la formación de placas calcificadas por depósito de cal-

1. Cirujano Plástico de la Clínica B\&S de Excelencia en Cirugía Plástica, Buenos Aires, Argentina.

2. Rotante de Cirugía Plástica de la Clínica B\&S de Excelencia en Cirugía Plástica, Buenos Aires, Argentina.

$\square$ Correspondencia: Dr. Guillermo Blugerman. Clínica B\&S de Excelencia en Cirugía Plástica. Laprida 1579. C1425EKK CABA, Rep. Argentina.drblugerman@centrosbys.com

Los autores no declaran conflictos de intereses

Recibido: 03/07/2018/Aceptado: 17/09/2018 cio en su superficie interna. Las cápsulas de las prótesis rugosas suelen ser macroscópicamente más gruesas y vascularizadas, en estas cápsulas, el depósito de fibras de colágeno es mayor, por lo que la cápsula aparenta ser más gruesa. Además, los implantes de superficie texturizada permiten que el tejido se introduzca en las irregularidades de la textura lo que hace que muchas veces haya un efecto velcro que adhiere el implante a los tejidos. La contractura capsular es la complicación más frecuente tras la cirugía de aumento mamario, aparece cuando la cápsula se engrosa y se contrae, comprimiendo al implante, deformándolo y alterando el resultado cosmético de la cirugía, creando disconfort a la paciente, malestar físico y psicológico. Las causas de aparición de una contractura capsular no son totalmente conocidas, pueden ser multifactoriales y aparecer luego de varios años de haberse realizado la cirugía, aunque la incidencia más importante se presenta en el primer año del posoperatorio. Sin embargo, se conocen factores que en cierto modo aumentarían la posibilidad de una contractura capsular, como la presencia de infección clínica y subclínica (Biofilm), hematomas, seromas, radioterapia administrada antes o después de la cirugía, entre otras causas. Cabe destacar que algunos estudios señalan la incidencia mayor de contractura capsular cuando el implante es colocado en plano subglandular. De acuerdo a la ASPS cerca de 400.000 mujeres de EE.UU. se realizan inserción de implantes mamarios rellenos de solución salina o de siliconas convirtiéndola en la cirugía más frecuente. Aunque ningún otro biomaterial revela propiedades comparables en términos de disponibilidad, adaptabilidad e inmunogenicidad, la silicona sigue siendo un cuerpo extraño propenso a una reacción fisiológica y obligatoria del cuerpo extraño. Por lo tanto, la silicona insertada se encapsula por el tejido fibrótico, que a pesar de la intención de proteger al organismo contra materiales potencialmente dañinos, en realidad puede conducir a una 


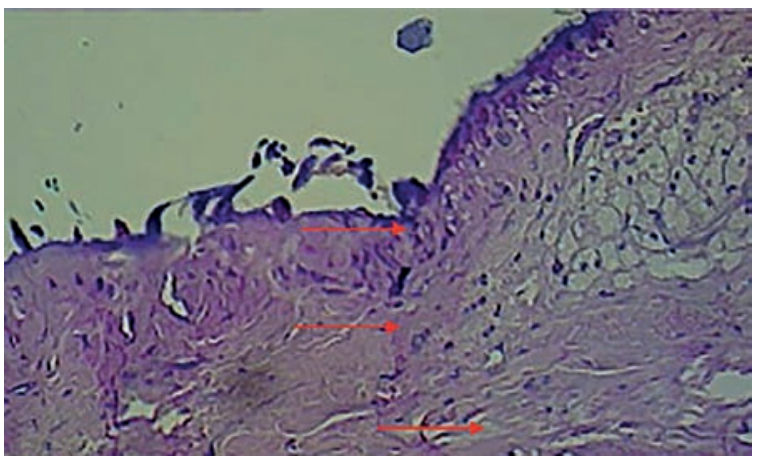

Figura 1.

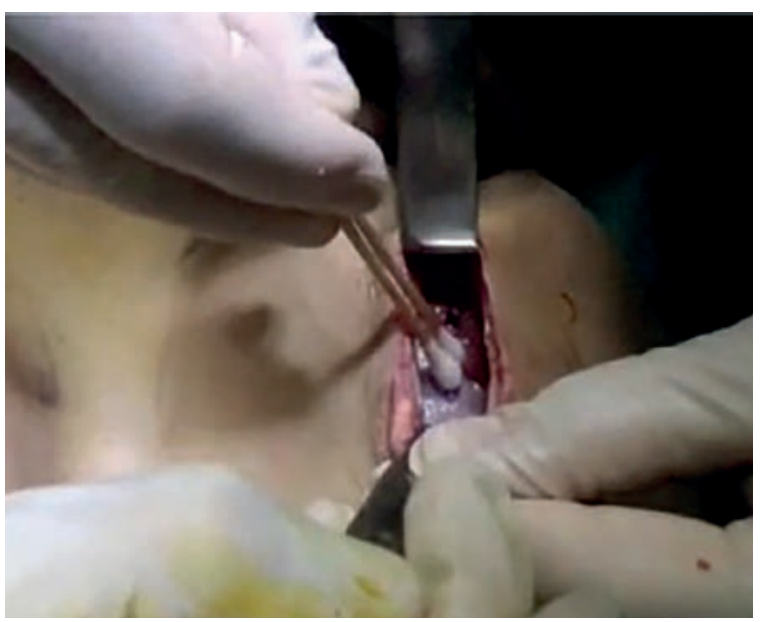

Figura 3.

complicación perjudicial en sí mismo, a saber, la contractura capsular. Las deformidades visibles, la dureza palpable y el dolor progresivo hacen que la contractura capsular sea clínicamente relevante en hasta un $30 \%$ de los casos cosméticos e incluso en el $73 \%$ de los casos reconstructivos después de la radioterapia; por lo tanto, la contractura capsular es la complicación a largo plazo más frecuente después de la reconstrucción y aumento con siliconas. Los mecanismos propuestos incluyen la inmunoestimulación directa y la infección subclínica, que se sostienen principalmente responsables de la iniciación y el mantenimiento de la formación de cápsulas. Ambos mecanismos son capaces de inducir una reacción inflamatoria crónica que estimula la proliferación y diferenciación de los fibroblastos y la consiguiente síntesis de colágeno y otras proteínas de la matriz extracelular. Considerando que las condiciones de trabajo estériles y antibióticos perioperatorios para reducir el riesgo de infección, las modificaciones del implante de silicona, especialmente su superficie, se inventaron para aumentar la biocompatibilidad y, por tanto, disminuir la fibrosis capsular. Hoy en día, están disponibles implantes de cubiertas lisas, texturados y poliuretanos. Aunque estos últimos revelaron buenos resultados en algunos estudios, la remoción puede ser dolorosa cuando es necesaria, y sobre todo en el contexto de la discusión en curso sobre la inducción del cáncer, los implantes cu-



Figura 2.

biertos con poliuretano son la opción terapéutica menos preferida para la mayoría de los cirujanos. En contraste, los implantes lisos o texturados se usan comúnmente en la práctica clínica para el aumento mamario. Curiosamente, la incidencia de la fibrosis capsular varía significativamente en la literatura actual y la elección del tipo de superficie se basa principalmente en la preferencia personal del cirujano. Sin embargo, en un metaanálisis publicado recientemente por Liu et al. solo las propiedades superficiales y en particular las superficies lisas están más probablemente asociadas con contractura capsular. Informes como este demuestran el impacto de la superficie del implante en la fibrosis capsular y la necesidad de nuevos estudios que puedan explicar estos hallazgos clínicos. Además, los modelos animales carecen de evaluaciones in vivo no invasivas de la formación de cápsulas y, especialmente, de métodos objetivos para evaluar la contractura, lo que es obligatorio para la investigación de aplicaciones terapéuticas en futuros estudios. El análisis histológico de las cápsulas periprotésicas mostró un patrón relativamente uniforme durante el período posoperatorio. Básicamente, se observaron tres capas alrededor de los implantes mamarios de silicona: una capa externa compuesta de tejido rico en colágeno, una capa interna correspondiente a la metaplasia similar a la sinovial; y una capa intermedia, predominantemente compuesta de tejido conjuntivo laxo (Figura 1). Las capas externa e intermedia estaban presentes en todos los casos. La eficacia de la texturización de las prótesis mamarias en la prevención de la contractura capsular ha sido reportada por más de 20 años. Se cree que las características de la superficie de la prótesis pueden causar cambios en el proceso de cicatrización. Una textura de menos de $150 \mathrm{~mm}$ de altura o profundidad suele conducir a la formación de una cápsula de tipo completo, mientras que un texturado más grueso e irregular $(200 \mathrm{~mm}$ de altura o profundidad) parece provocar una cápsula irregular e interrumpida con el crecimiento del tejido, lo que conduce a un mayor efecto de unión, que se ha relacionado con la reducción no sólo de la inflama- 

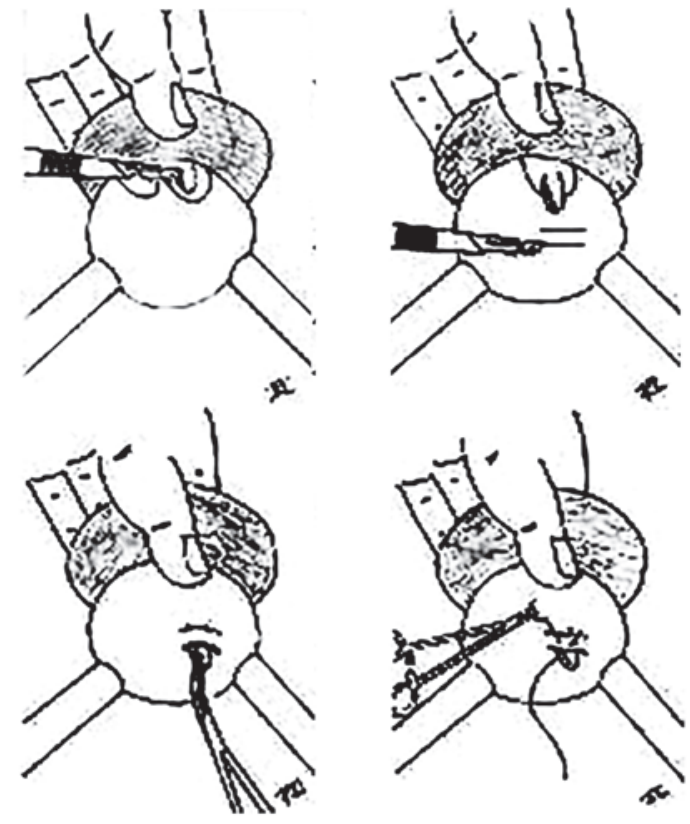

Figura 4.

ción sino también de la contractura capsular. Por el contrario, algunos investigadores creen que la superficie de los implantes mamarios texturados y sus cápsulas pueden no estar directamente relacionadas con la prevención de la contractura capsular. Según Burkhardt et al. ${ }^{1}$ (1981) y Virden et al. ${ }^{3}$ (1992), una respuesta tisular que implica una prótesis y acompaña sus contornos es una respuesta fisiológica esperada de curación a material extraño; además, esta respuesta puede resultar en contractura capsular con la consecuente modificación de la forma y consistencia de la mama. Estos efectos son eventos indeseables en procedimientos de cirugía plástica. La contractura capsular parece tener un efecto idiosincrático en los seres humanos. Puede ocurrir unilateralmente y en diferentes períodos posoperatorios. Además, generalmente ocurre dentro de los primeros años después del aumento mamario. Considerando que la contractura capsular puede ser el resultado de una respuesta progresiva y de un tejido de reparación causada por la presencia de un cuerpo extraño, decidimos evaluar el proceso de cicatrización por un período de hasta 9 meses. Por lo tanto, con el fin de mejorar la comprensión del fenómeno de la curación después del aumento mamario, la intensidad de la contractura capsular se controló mediante el método de tonometría de aplanación. Los valores encontrados se pudieron correlacionar con el análisis histológico durante el período posoperatorio considerando la influencia de las cubiertas de implantes lisas o texturizadas. En nues-

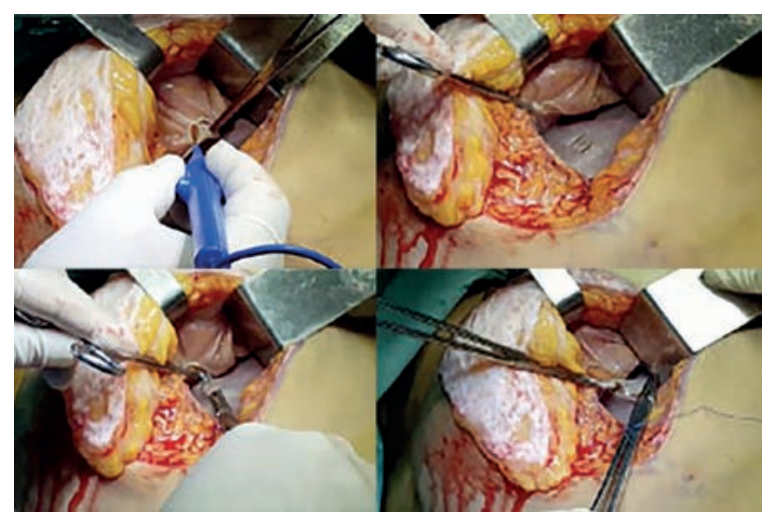

Figura 5.

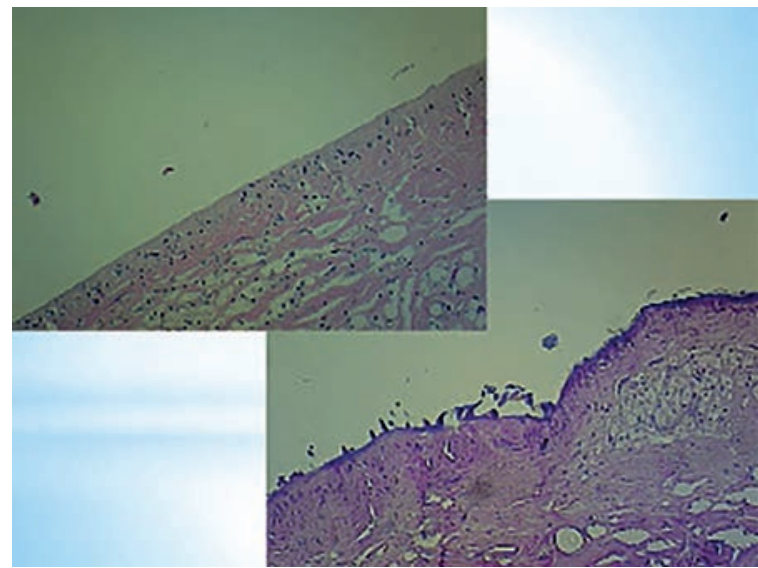

Figura 6.

tro estudio, la metaplasia tipo sinovial ocurrió más a menudo en los implantes texturados, lo que indica que este factor también puede estar relacionado con la disminución de la aparición de contractura capsular después del aumento mamario. Es importante mencionar que el papel de estas capas no colagenosas en la contracción capsular es todavía desconocido y merece un estudio más extenso para una mejor comprensión de este evento. En ambos grupos, el grosor de la capa de colágeno no presentó variaciones significativas durante los períodos evaluados (todos los factores mostraron valores descriptivos. Por lo tanto, en ambos grupos, se produjo una variación en el espesor total de la cápsula debido al aumento de la No colagante, compuesta de tejido conjuntivo y, a veces, de metaplasia sinovial.

\section{MATERIAL Y MÉTODO}

En las situaciones que requieren la extracción definitiva del implante, la extracción transitoria del mismo o el cambio de la posición del implante del plano retromamario a retromuscular y viceversa, citando evidencia de que las cápsulas relacionadas con implantes mamarios se resuelven sin incidentes, los cirujanos han elegido dejar las cápsulas en su lugar cuando se retiran los implantes porque la capsulectomía agrega tanto la morbilidad como el gasto al procedimiento. Sin 


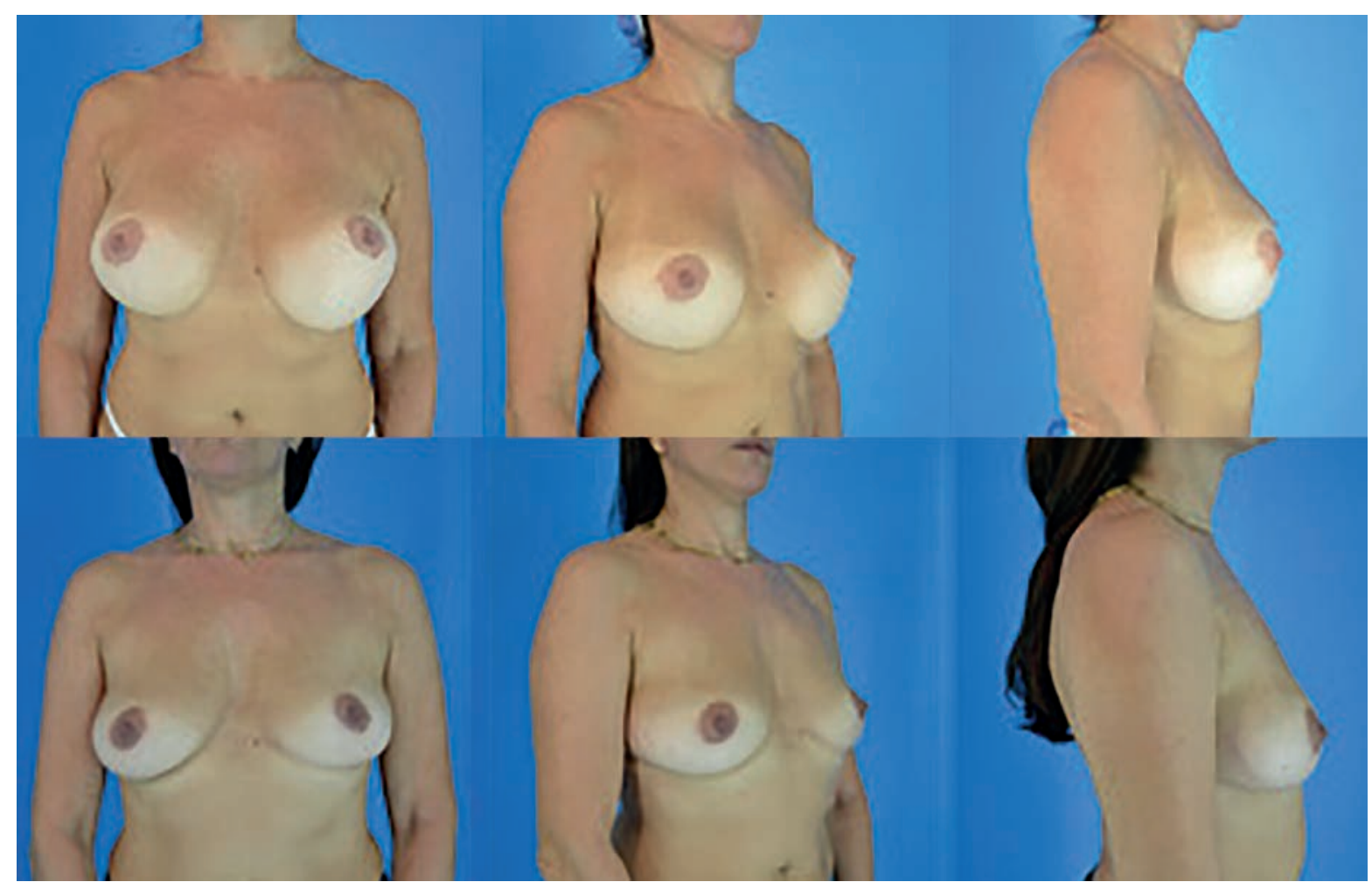

Figura 7.

embargo, la evidencia clínica e histopatológica reciente sugiere que la resolución sin complicaciones no siempre es el caso, y varios problemas potenciales pueden surgir de cápsulas retenidas después de la eliminación del implante. Las cápsulas de implante retenidas pueden resultar en una masa espiculada sospechosa de carcinoma, calcificaciones densas que oscurecen el tejido mamario vecino en estudios posteriores de imágenes y masas quísticas debidas a derrame seroso persistente, hematoma expansivo o quistes rellenos de silicona encapsulados. Además, las cápsulas retenidas son un depósito de material extraño relacionado con el implante en el caso de implantes rellenos de gel de silicona e implantes texturados que promueven el crecimiento del tejido. En estos casos se puede recurrir a la capsulectomía total, parcial o al abandono de la cápsula vacía. En otras situaciones se puede requerir un tratamiento parcial de la cápsula para reacomodar un implante desplazado patológicamente que requiere la realización de una capsulorrafia, capsulotomía o capsulectomía.

Ante la presencia de cápsula residual, ¿̇cuáles son las opciones?

- Capsulectomía quirúrgica total o parcial. Las desventajas de la capsulectomía en el momento de la remoción del implante incluyen el aumento del tiempo operatorio y la pérdida de sangre. Los implantes retroglandulares (submamarios) desarrollan cápsulas que se adhieren fuertemente a la fascia pectoral y los músculos pectorales bien vascularizados, mientras que submuscular (subpectoral) puede adherirse a las costillas y a los músculos intercostales.
Para prevenir el hematoma y disminuir el riesgo de neumotórax, algunos cirujanos extraen la pared anterior de la cápsula, dejando la cápsula posterior en su lugar en la pared torácica.

- Capsulorrafia con suturas. Se recurre a suturas que unen la cápsula anterior con la parietal al estilo de los puntos de Baroudi utilizados en las abdominoplastia para reducir el riesgo de formación de seromas. Cuando de realiza esta técnica se deja abandonada la pseudosinovia que recubre la cara interna de las cápsulas y no se inactiva el biofilm existente en los tejidos. Pocos autores han abordado las consecuencias de las cápsulas retenidas. Se han descrito masas mamarias palpables o detectadas mamográficamente relacionadas con cápsulas de implantes mamarios. Tales masas pueden estar bien circunscritas o bilateralmente simétricas, lo que indica su naturaleza benigna y a menudo no se han extirpado. Las masas espiculadas, por el contrario, son extirpadas para el examen histopatológico para descartar el adenocarcinoma.

- Capsulotomía radiada. Este método busca la destrucción de la cavidad dejando abandonadas porciones de cápsula con sus correspondientes pseudosinovias en contacto con los tejidos. No se ha determinado que sucede con estos tejidos después de su abandono. El procedimiento suele ser sangrante y puede producir la formación de hematomas de mayor o menor cuantía. Estos hematomas presentan un mayor riesgo de infección debido a la presencia de biofilm sobre el tejido y de fibrosis residual.

- Nosotros proponemos una nueva opción, la Capsu- 


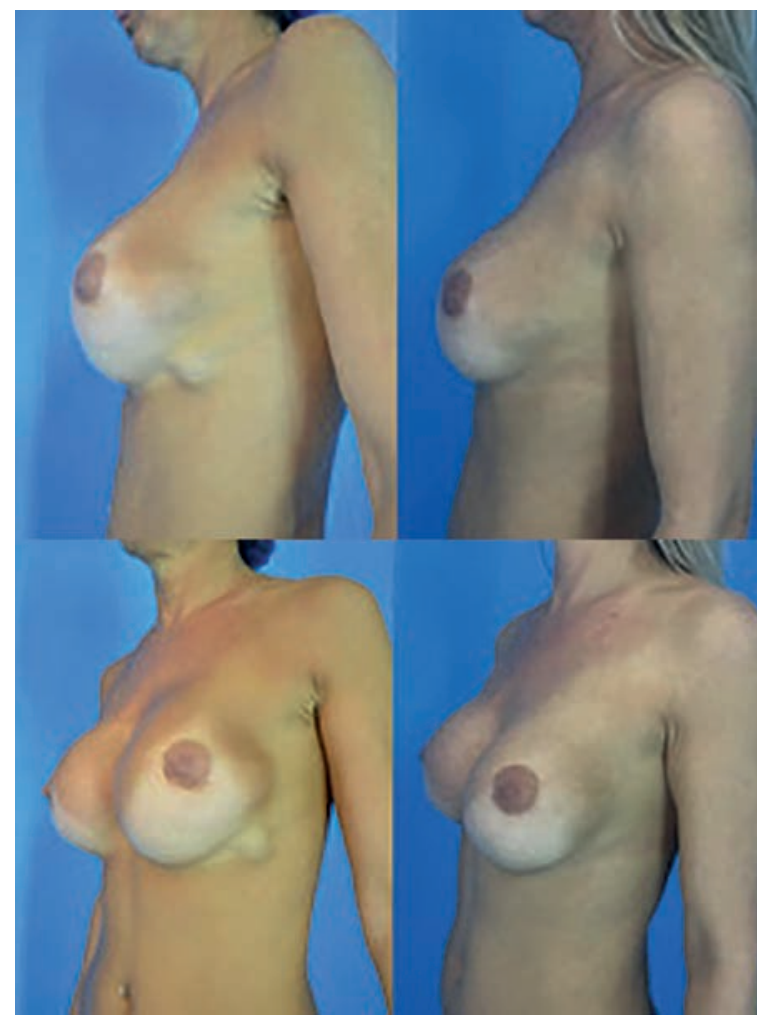

Figura 8.

lectomía química total o parcial y cierre de la cavidad con colgajos de anclaje.

Como ha sido demostrado histológicamente, la superficie interior de las cápsulas mamarias, presentan una metaplasia epitelial similar a la sinovia de las articulaciones que suele ser de carácter exudativo. Además en muchos de los casos se observa la presencia de residuos de siliconas que en cierto grado impermeabilizan la cápsula. Las otras capas de la cápsula no son otra cosa que estructuras fibrocelulares de tejido de reacción hacia el cuerpo extraño. Si quisiéramos lograr el colapso de esta cavidad deberíamos destruir solo la capa interna de la misma. Para lograrlo se pueden utilizar métodos físicos, mecánicos o químicos. Entre los métodos físicos podríamos imaginar el uso de un láser ablativo como el de $\mathrm{CO}_{2}$ o el de erbio, o utilizar alguna droga de terapia fotodinámica, pero consideramos que serían métodos más caros y más complicados en su aplicación. En los métodos mecánicos podríamos utilizar una abrasión mecánica de la cara interna de la cápsula, pero por las características de este tejido y su ubicación pareciera ser poco viable con los elementos que contamos hoy en día. Finalmente nos queda la posibilidad de la utilización de un método químico. Entre los elementos más utilizados en la medicina se encuentran los ácidos que son líquidos cáusticos de amplio empleo en dermatología para eliminar las capas superiores de la piel. Entre los distintos ácidos, hay uno que es muy eficiente, de bajo costo y que produce un efecto de frosting



Figura 9.

que facilita la identificación del área tratada (Figura 2). Basados en nuestra experiencia en el uso del ácido tricloroacético (TCA) en lesiones de acné mediante la técnica CROSS, y habiendo observado la alta eficiencia que tiene este ácido para destruir los tejidos epiteliales, y su ausencia de toxicidad local y sistémica decidimos emplearlo con el objeto de realizar una capsulectomía química. Para lograr una acción ablativa rápida, efectiva y con efecto autolimitante fue que decidimos la concentración de $90 \%$.

\section{TÉCNICA QUIRÚRGICA}

Una vez retirado el implante, se procede a la limpieza de la cavidad con gasas para remover toda secreción y doble cápsula que pudiera existir. Luego se tópica toda la cara interna de la cavidad utilizando para ello hisopos de algodón estériles, embebidos con la solución de TCA al 90\% (Figura 3). Inmediatamente al tomar contacto los tejidos con el ácido se produce una apoptosis y muerte celular de las capas pseudosinoviales, lo que se manifiesta por una coloración blanca nacarada de los tejidos. Existiría la posibilidad de utilizar un colorante azul como en la técnica del peeling de Obagi para asegurarnos de haber tratado la totalidad de la superficie capsular interna.

Se espera dos minutos para que el ácido actúe completamente y se lo neutraliza con la instilación en la cavidad de 30 o $40 \mathrm{ml}$ de bicarbonato molar y abundante solución salina estéril. Una vez terminado el proceso de ablación química y con el fin de poder predecir la posición del tejido remanente mamario promoviendo una adherencia de los tejidos en la posición deseada por el cirujano, complementamos la ablación con la realización decolgajos capsulares de anclaje. Es un colgajo que a modo de llave-cerradura permite una mejor coaptación de las dos paredes capsulares, cerrando el espacio muerto y permitiendo además la elevación y fijación de los tejidos mamarios, dando un efecto de pexia interna (Figuras 4, 5). En los casos de capsulectomía química para cambio de plano del implante o de explantación definitiva dejamos un drenaje no aspira- 
tivo consistente en una Angiocath 14 modificado. En los caso de capsulectomía química para el tratamiento del seroma tardío o recidivante dejamos un drenaje espirativo por contrabertura.

\section{HISTOLOGÍA}

Se enviaron muestras para su estudio anatomopatológico y se compararon los aspectos de la pseudosinovia antes y después de la topicación con TCA. En la cápsula sin tratar, las secciones muestran tejido fibroconectivo en el cual se reconoce cápsula con metaplasia sinovial compuesta por células fibrohistiociticas. Las secciones tratadas con TCA muestran tejido fibroconectivo en el cual se reconoce cápsula con metaplasia sinovial; esta presenta menor espesor con respecto a la muestra control. Se observa menor número de células, acidofilia del citoplasma y presencia de proyecciones "digitiformes" en la superficie (hacia la cavidad). En algunos sectores se observa pérdida de la capa de células fibrohistiociticas que componen la membrana sinovial (Figura 6).

\section{INDICACIONES DE ESTA TÉCNICA}

Explantación definitiva (Figura 7). Explantación transitoria. Tratamiento del seroma perimplante (Figura 8). Remodelación parcial de la cápsula para corregir sinmastia (Figura 9). Remodelación parcial de la cáp- sula para corregir desplazamiento axilar del implante. Remodelación parcial de la cápsula para corregir bottoming up. Cambio de plano del implante desde el subpectoral al retroglandular. Cambio de plano del implantes desde el retroglandular al subpectoral.

\section{CASUÍSTICA}

Hemos utilizado esta metodología en 61 pacientes para tratar las distintas opciones en las que ha sido necesario actuar sobre una cápsula residual.

Explantación definitiva, 10 pacientes. Explantación transitoria por extrusión, 2. Tratamiento del seroma periimplante, 2. Remodelación parcial de la cápsula para corregir sinmastia, 2. Remodelación parcial de la cápsula para corregir desplazamiento axilar del implante, 10 . Remodelación parcial de la cápsula para corregir bottomingup, 4. Cambio de plano del implante desde el subpectoral al retroglandular, 1. Cambio de plano del implante desde el retroglandular al subpectoral. 30.

\section{CONCLUSIONES}

Los resultados obtenidos con esta metodología han sido altamente satisfactorios para corregir los problemas que presentaban las pacientes y la prevención de la aparición de complicaciones que pudieran surgir al dejar las cápsulas abandonadas a su libre evolución.

\section{BIBLIOGRAFÍA}

1. Fischer S, Hirche C, Reichenberger MA, Kiefer J, Diehm Y, Mukundan SJr, et al. Silicone Implants with Smooth Surfaces Induce Thinner but Denser Fibrotic Capsules Compared to Those with Textured Surfaces in a Rodent ModelPLOS ONE / DOl:10.1371/journal.pone.0132131 July 7, 2015

2. Kasper CS. Histologic features of breast capsules reflect surface con $\mathrm{fi}$ guration and composition of silicone bag implants. Am J Clin Pathol 102: 655, 1994.

3. Bucky LP, Ehrlich HP, SohoniS, et al. The capsule quality of saline-filled smooth silicone, textured silicone, and polyurethane implants in rabbits: A long-term study. Plast Reconstr Surg 1994;93:1123.

4. Collis N, Coleman D, Foo IT, et al. Ten-year review of a prospective randomized controlled trial of textured versus smooth subglandular silicone gel breast implants. Plast Reconstr Surg 106: 786, 2000.

5. Burkhardt BR, Dempsey PD, Schnur PL, et al. Capsular contracture: A prospective study of the effect of local antibacterial agents. Plast Reconstr Surg 77: 919, 1986.

6. Luke JL, Kalasinsky VF, Turnicky RP, et al. Pathological and biophysical findingsassociated with silicone breast implants: A study of capsular tissues from 86 cases. Plast. Reconstr. Surg. 100: 1558, 1997.

7. Elisa Minamiet all. The Composition and Behavior of Capsules around Smooth and Textured Breast Implants in Pigs Plastic and Reconstructive Surgery • September 15, 2006

8. Bern S, Burd A, May JW. The biophysical and histologic properties of capsulesformed by smooth and textured silicone implants in the rabbit. Plast Reconstr Surg 89:1037, 1992.
9. Brodland DG, Roenigk RK, Cullimore KC, Gibson LE. Depths of chemexfoliation induced by various concentrations and application techniques of trichloroacetic acid in aporcine model. J Dermatol Surg Oncol 1989;15:967-71.

10. El-Domyati MB, Attia SK, Saleh FY, et al. Trichloroacetic acid peeling versusdermabrasion: a histometric, immunohistochemical, and ultrastructural comparison. Dermatol Surg 2004;30: 179-88.

11. Rockwell BW, Casey HD, Cheng CA. Breast capsule persitence after breast implant removal. Plastic and Reconstructive Surgery: April 1998 - Volume 101 - Issue 4 - pp 1085-1088.

12. Marques M, Brown S, Correia-Sá IM, Cordeiro NDS, Rodrigues-Pereira P, Gonçalves Rodrigues A, Amarante J. The Impact of Triamcinolone Acetonide in Early Breast Capsule Formation in a Rabbit Model. Aesthetic Plastic Surgery:August 2012, Volume 36, Issue 4, pp 986-94.

13. Kim YS, Hong JW, Yoon JH, et al. Botulinum Toxin Affects Early Capsule Formation Around Silicone Implants in a Rat Model. Annals of Plastic Surgery: April 2015 - Volume 74 - Issue 4 - p 488-495.

14. Rohrich RJ, Beran SJ, Restifo RJ, Copit S. Aesthetic Management of the Breast following Explantation: Evaluation and Mastopexy Options. Plastic and Reconstructive Surgery: March 1998 - Volume 101 - Issue 3 - ppg 827-837. 